\title{
Simulation of multi-pulse coaxial helicity injection in the Sustained Spheromak Physics Experiment
}

J. B. O'Bryan, C. A. Romero-Talamás, and S. Woodruff

Citation: Physics of Plasmas 25, 032503 (2018); doi: 10.1063/1.5018319

View online: https://doi.org/10.1063/1.5018319

View Table of Contents: http://aip.scitation.org/toc/php/25/3

Published by the American Institute of Physics

\section{Articles you may be interested in}

The physical foundation of the reconnection electric field

Physics of Plasmas 25, 032901 (2018); 10.1063/1.5021461

Magnetic plasma expulsion

Physics of Plasmas 25, 012508 (2018); 10.1063/1.5006887

Editorial: Preface to the 25th Volume of Physics of Plasmas

Physics of Plasmas 25, 010401 (2018); 10.1063/1.5021964

Stabilization of a magnetic island by localized heating in a tokamak with stiff temperature profile

Physics of Plasmas 25, 022514 (2018); 10.1063/1.5021759

Poloidal asymmetries of flows in the Tore Supra tokamak

Physics of Plasmas 25, 020704 (2018); 10.1063/1.5022122

Algebraic motion of vertically displacing plasmas

Physics of Plasmas 25, 022516 (2018); 10.1063/1.5011176

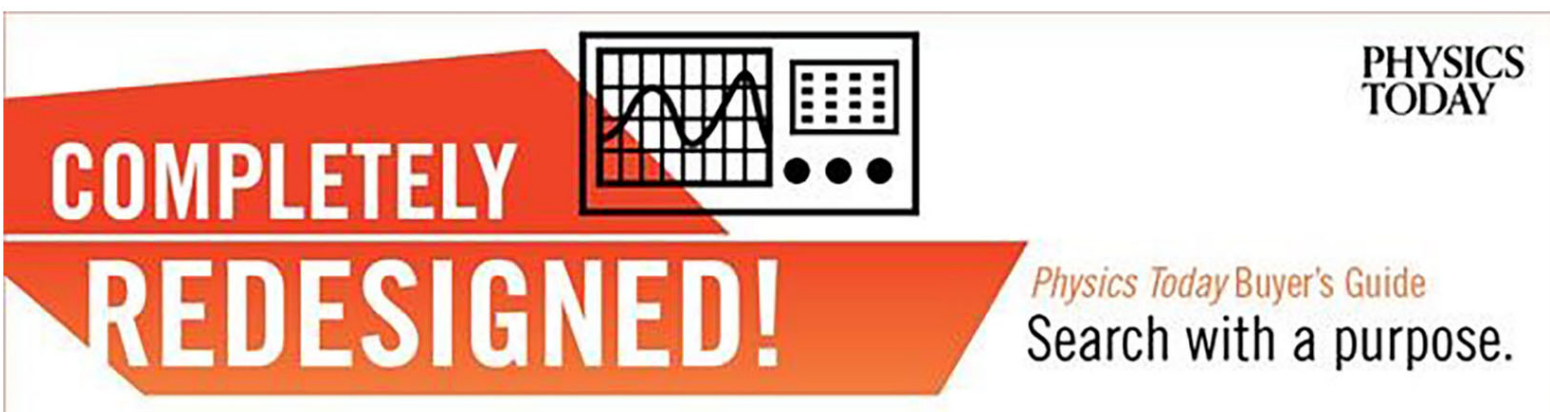




\title{
Simulation of multi-pulse coaxial helicity injection in the Sustained Spheromak Physics Experiment
}

\author{
J. B. O'Bryan, ${ }^{1, a)}$ C. A. Romero-Talamás, ${ }^{1}$ and S. Woodruff ${ }^{2}$ \\ ${ }^{1}$ Department of Mechanical Engineering, University of Maryland, Baltimore County, Baltimore, \\ Maryland 21250, USA \\ ${ }^{2}$ Woodruff Scientific, Inc., Seattle, Washington 98103, USA
}

(Received 5 December 2017; accepted 13 February 2018; published online 1 March 2018)

\begin{abstract}
Nonlinear, numerical computation with the NIMROD code is used to explore magnetic selforganization during multi-pulse coaxial helicity injection in the Sustained Spheromak Physics eXperiment. We describe multiple distinct phases of spheromak evolution, starting from vacuum magnetic fields and the formation of the initial magnetic flux bubble through multiple refluxing pulses and the eventual onset of the column mode instability. Experimental and computational magnetic diagnostics agree on the onset of the column mode instability, which first occurs during the second refluxing pulse of the simulated discharge. Our computations also reproduce the injector voltage traces, despite only specifying the injector current and not explicitly modeling the external capacitor bank circuit. The computations demonstrate that global magnetic evolution is fairly robust to different transport models and, therefore, that a single fluid-temperature model is sufficient for a broader, qualitative assessment of spheromak performance. Although discharges with similar traces of normalized injector current produce similar global spheromak evolution, details of the current distribution during the column mode instability impact the relative degree of poloidal flux amplification and magnetic helicity content. Published by AIP Publishing.

https://doi.org/10.1063/1.5018319
\end{abstract}

\section{INTRODUCTION AND MOTIVATION}

The spheromak is an alternative confinement concept with many applications that benefit basic plasma research and the mainline fusion program. In particular, the spheromak has proven to be a robust tool for studying magnetic self-organization in parameter regimes relevant to both astrophysical and fusion plasmas. ${ }^{1}$ The Sustained Spheromak Physics eXperiment (SSPX) achieved encouraging results during its operation, including peak electron temperature $T_{e}$ $\sim 0.5 \mathrm{keV}$, toroidal magnetic field on axis $B_{\text {tor }}>1 \mathrm{~T}$, plasma current $I_{p} \sim 1 \mathrm{MA}$, and peak $\beta_{e}>5 \%$, while being limited by its power system and heat dissipation capabilities and not by some physical process inherent to its operation. ${ }^{2}$ Despite its promise as a confinement concept, the spheromak has only been explored at the basic plasma science and concept exploration levels.

In addition to being able to achieve the high masspower-density required for thermonuclear fusion, the spheromak confinement concept possesses several advantages that not only makes it suitable for a compact neutron source but also uniquely suitable for university-scale experimentation. Spheromaks typically lack a central conductor, which allows for more compact design. Unlike the two leading magnetic confinement concepts - the tokamak and stellarator-the dominant component of the magnetic field in a spheromak is generated by internal plasma currents, as opposed to external field coils. ${ }^{3}$ The required vacuum magnetic field strengths

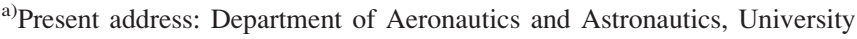
of Washington, Seattle, Washington 98195, USA. Electronic mail: jobryan@uw.edu.
}

are easily achievable using copper magnets, which negates the need for more costly superconducting magnets and their associated cryogenic cooling systems. These relaxed engineering requirements result in lower capital costs for a new experiment compared to other confinement concepts.

Our underlying goal is to develop the spheromak concept into a compact, pulsed fusion neutron source. We are considering two approaches for sustaining and heating a spheromak plasma to fusion temperatures: multi-pulse coaxial helicity injection (CHI) in this paper and magnetic compression in Ref. 4. For both approaches, the initial spheromak plasma is formed by CHI. While a spheromak could achieve quasisteady-state operation through external heating and current drive, e.g., neutral beam injection ${ }^{5}$ or imposed dynamo current drive, ${ }^{6}$ it would increase the size, engineering complexity, and capital costs associated with an experiment. With either approach, significant theoretical, computational, and engineering developments are still required to advance to a full-fledged proof-of-principle device capable of confining fusion plasmas for times long enough to test alpha particle heating and key engineering issues.

In this paper, we explore multi-pulse operation ${ }^{7}$ of SSPX using nonlinear, numerical computation. Multi-pulse operation seeks to sustain a spheromak plasma against resistive decay by repeatedly pulsing the coaxial gun current above the threshold for poloidal flux amplification. Previous numerical studies ${ }^{8-10}$ of the SSPX spheromak-even those considering the implications of refluxing - only simulated a single injector pulse cycle, i.e., only the first injector current pulse produced poloidal flux amplification. Additional injector pulses, if present, were solely used for sustaining the 
spheromak plasma, not for producing additional poloidal flux amplification. The motivation for these earlier studies was to provide theoretical support for the column-mode instability as the mechanism for the change in magnetic topology to a configuration with more favorable confinement properties and to explain the experimentally observed electron transport.

Here, we simulate the continuous evolution of multipulse spheromaks to understand the effect of refluxing and find ways to qualitatively improve spheromak performance. Achieving our ultimate goal of designing a future spheromak experiment will, by necessity, require a much broader exploration of spheromak formation and sustainment than the multi-pulse cases presented in this study. By determining the simplest and most computationally efficient numerical model capable of qualitatively assessing spheromak performance, we can minimize the computational expense of our calculations, which will allow us to explore a greater number of candidate geometries and modes of operation.

In Sec. I A, we provide a theoretical description of the column mode instability, the mechanism which produces change in the magnetic topology and the resulting poloidal flux amplification necessary for high performance spheromak operation. Then, we describe the physical parameters and numerical model evolved in our computations in Sec. II. In Sec. III, we simulate entire multi-pulse discharges in SSPX, describing the plasma evolution over multiple pulses of injector current. We employ multiple thermal conduction models to demonstrate the robustness of the global spheromak evolution.

\section{A. Spheromak evolution}

The formation and sustainment of a spheromak with coaxial helicity injection (CHI) relies on driven magnetic self-organization. Prior to current drive, the initial vacuum magnetic field is allowed to equilibrate through the conducting vacuum vessel. ${ }^{11}$ Hence, the terms vacuum, bias, and soaking flux are often used interchangeably. During current drive, two electrodes connected by the vacuum magnetic field are biased relative to each other. Current flows between the electrodes, and the resulting Lorentz force causes expansion of the injector flux bubble into the flux conserver, a close-fitted, conducting vacuum vessel.

The evolution of the spheromak is defined by two threshold conditions on the injector current. First, the injector current must exceed some threshold for the ejection of the injector flux bubble into the flux conserver. In SSPX, this was referred to as the bubble burst criterion. ${ }^{12}$ This threshold is determined by the bias flux configuration. The experimental shots modeled in this study employed the modified flux (MF) configuration for the vacuum magnetic field in SSPX, shown in Fig. 1. In this configuration, the magnetic field strength decreases more smoothly from the tail to the mouth of injector, i.e., towards the flux conserver, which results in a lower, less well defined threshold current for the bubble burst criterion. The magnetic flux also extends further into the flux conserver.

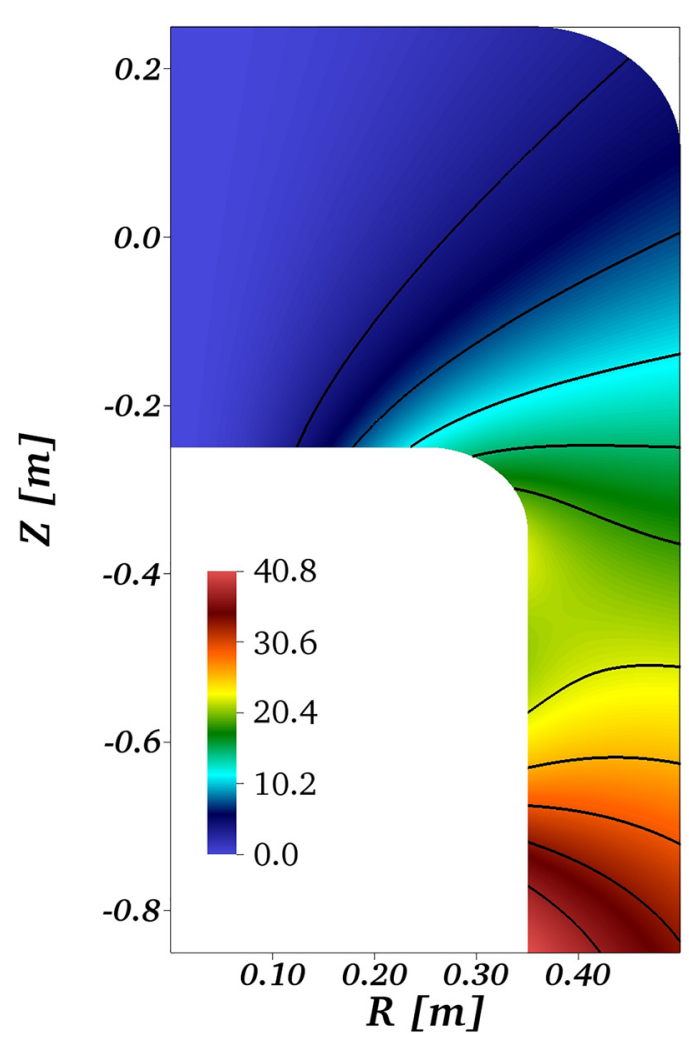

FIG. 1. Our computations start from vacuum magnetic fields corresponding to specific experimental discharges in the SSPX spheromak. Color contours show the poloidal magnetic flux profile in units of $\mathrm{mWb}$ consistent with SSPX shot \#19719, i.e., $40 \mathrm{mWb}$ of nominal magnetic flux in the modified flux (MF) configuration. Black contour lines are shown at equally spaced contour levels to highlight the flux profile shape in the injector region.

After reaching the ejection threshold, the injector flux bubble eventually fills the flux conserver. The injector current flows around the flux bubble producing an annular current column near the geometric axis. Once the injected current exceeds the second threshold condition, it excites an $n_{\phi}=1$ kink instability of the current column that changes the magnetic topology: The current column bends and reconnects forming a loop of current near the mid-plane. ${ }^{13}$ This process is phenomenologically similar to current loops formed during localized helicity injection in the Pegasus spherical tokamak. ${ }^{14}$ For the SSPX and SPHEX spheromaks, this instability was referred to as the "column mode" and "dough hook" instability, respectively.

The column mode acts as a semi-coherent dynamo that converts predominantly poloidal edge current into toroidal current near the magnetic axis, or equivalently, converting toroidal flux from the injector into poloidal flux. ${ }^{15}$ Like with current loops formed in the Pegasus ST, the dominantly $n_{\phi}=1$ perturbations of the velocity and magnetic field beat together to produce a net toroidally averaged electric field transferring energy to the mean $\left(n_{\phi}=0\right)$ magnetic field. ${ }^{16}$ Poloidal flux amplification in a spheromak is also phenomenologically equivalent to toroidal field reversal in a Reversed Field Pinch (RFP) — though typically more extreme-with the $r-z$ plane in a spheromak corresponding to the $r-\phi$ plane in an RFP. ${ }^{17}$

Linear, ideal MHD stability analysis predicts the onset of the column mode instability in terms of the core, edge, 
and the eigenvalue (Taylor state) $\lambda$ 's, where $\lambda=\mu_{0} J_{\|} / B .{ }^{18}$ However, this theory does not directly address the accessibility of the equilibria or poloidal flux amplification, which are determined by nonlinear plasma evolution. The instability is self-stabilizing, as the accumulation of poloidal flux effectively reduces the value of $\lambda$. Resistive decay of the toroidal current near the magnetic axis increases $\lambda$, which once again triggers the instability and further toroidal current drive. Coaxial helicity injection acts as a strong source of edge current drive, meaning that prior to the column mode instability and poloidal flux amplification, the core $\lambda$ is negligible for all spheromaks formed with CHI. However, the toroidal current drive produced during the column mode instability means that the core $\lambda$ will be non-negligible during refluxing, i.e., when driving an additional pulse of injector current. Therefore, the threshold for the column mode instability will change during the course of multi-pulse operation.

The highest temperatures are typically observed during relaxation of a spheromak plasma, when the injector current is reduced after the column mode instability. ${ }^{10,11}$ The column mode is necessary for poloidal flux amplification, which is in turn necessary for good thermal confinement in the relaxing spheromak. During relaxation, non-axisymmetric perturbations of the magnetic field decay more rapidly than the amplified symmetric poloidal flux. ${ }^{17}$ Consequently, closed magnetic surfaces form over a significant fraction of the plasma volume, aided by the toroidal current produced by the column mode instability and reduction of the injector current, which perturbs the edge magnetic field. Thermal transport occurs at a much slower rate across these closed magnetic surfaces.

\section{NUMERICAL MODELING}

In the following study, we model three-dimensional spheromak evolution with the NIMROD code, ${ }^{19}$ starting from vacuum magnetic fields and progressing through multiple distinct phases. These computations use resistive, fluidbased modeling with realistic, evolving, locally computed transport coefficients-particularly thermal conductivity and resistivity-and ohmic heating. The model equations are listed as follows:

$$
\begin{gathered}
\frac{\partial n}{\partial t}+\nabla \cdot(n \mathbf{v})=\nabla \cdot\left(D_{n} \nabla n\right) \\
\rho\left(\frac{\partial \mathbf{v}}{\partial t}+\mathbf{v} \cdot \nabla \mathbf{v}\right)=\mathbf{J} \times \mathbf{B}-\nabla p+\nabla \cdot\left(\rho \nu_{i s o} \mathbf{W}\right) \\
\frac{n k_{B}}{\gamma-1}\left(\frac{\partial T_{s}}{\partial t}+\mathbf{v}_{s} \cdot \nabla T_{s}\right)=-n k_{B} T_{s} \nabla \cdot \mathbf{v}_{s}-\nabla \cdot \mathbf{q}_{s}+Q_{s} \\
\frac{\partial \mathbf{B}}{\partial t}+\nabla \times(\eta \mathbf{J}-\mathbf{v} \times \mathbf{B})=\kappa_{\nabla \cdot \mathbf{B}} \nabla \nabla \cdot \mathbf{B} \\
\mu_{0} \mathbf{J}=\nabla \times \mathbf{B}
\end{gathered}
$$

where the subscript $s$ indicates particle species and $\mathbf{W}$ is the traceless rate-of-strain tensor. A similar model has been used to study the interaction between thermal transport and magnetic relaxation in previous numerical studies of the SSPX spheromak. 9,20,21 However, as described below, our model includes some key differences in the transport model and the implementation of the injector gap.

The initial condition of our SSPX simulations is treated as plasma, albeit at an unphysically low temperature of $0.125 \mathrm{eV}$. The precise value of the initial temperature-which is also used as the fixed boundary temperature-is not critical, merely that the temperature is cold enough that the walls act as an effective thermal sink for parallel heat transport along open field lines. The electron number density is uniformly set to $1 \times 10^{20} \mathrm{~m}^{-3}$ across the entire domain. We do not consider ionization energy in our modeling. As the transition from isotropic $\left(\omega_{c i} \tau_{i} \ll 1\right)$ to anisotropic $\left(\omega_{c i} \tau_{i} \gg 1\right)$ thermal conduction may be important as the local plasma temperature increases, we employ the Braginskii formulation $^{22}$ for our transport model coefficients. This thermal conduction model has also been used to study non-inductive startup in the Pegasus spherical tokamak. ${ }^{14}$

The general form for the heat flux is given by Eq. (6). The single temperature model uses a single heat flux, which employs the larger parallel electron $\left(\chi_{\|}=\chi_{\|, e} \gg \chi_{\|, i}\right)$ and perpendicular ion $\left(\chi_{\perp}=\chi_{\perp, i} \gg \chi_{\perp, e}\right)$ thermal diffusivities for the transport coefficients. The two temperature model uses separate ion $\left(\mathbf{q}_{i}\right)$ and electron $\left(\mathbf{q}_{e}\right)$ heat fluxes, which employ separate parallel and perpendicular thermal diffusivities for each species. The sole source of volumetric heating is resistive dissipation, $Q=\eta J^{2}$. In addition to resistive heating, which is limited to the electrons, the two-temperature model includes temperature-dependent thermal equilibration between electrons and ions, given by Eqs. (7) and (8), where $\sigma$ is the rate of thermal equilibration

$$
\begin{gathered}
\mathbf{q}_{s}=n k_{B}\left[\left(\chi_{\|, s}-\chi_{\perp, s}\right) \mathbf{b b}+\chi_{\perp, s} \mathbf{I}\right] \cdot \nabla T_{s}, \\
Q_{i}=n_{e} k_{B}\left(T_{e}-T_{i}\right) \frac{\sigma}{\gamma-1}, \\
Q_{e}=\eta J^{2}-n_{e} k_{B}\left(T_{e}-T_{i}\right) \frac{\sigma}{\gamma-1} .
\end{gathered}
$$

In both sets of calculations, the time step is limited by the Courant-Friedrichs-Lewy condition. ${ }^{23}$ However, the limiting flow velocity is different for the two sets. Whereas the singletemperature cases are limited by the center-of-mass flow velocity, $\mathbf{v}$, the two-temperature cases are limited by the, typically much faster, electron flow velocity, $\mathbf{v}_{e}=\mathbf{v}-(n e)^{-1} \mathbf{J} /$ $\left(1+Z_{\text {eff }} m_{e} / m_{i}\right)$. Assuming that both sets of calculations produce the correct magnetic evolution, the single-temperature case will require many fewer CPU-hours to simulate the same amount of physical time.

Initially, the calculations employ a no-flux boundary condition for the particle density across the entire domain boundary. When the particle density across the injector gap drops to a specified value-typically $1 \%-2 \%$ of the initial value-it is then held fixed at that value. This implementation allows the flow to become realistically choked, while avoiding numerical issues associated with extremely large density gradients. It also relaxes the need for artificial particle diffusivity.

Like the previous numerical studies ${ }^{8-10}$ of SSPX, the injected current is specified by setting $R B_{\phi}=\mu_{0} I_{i n j} / 2 \pi$ 
across the injector gap. The injector voltage is computed from the plasma model by integrating the resistive and MHD electric fields across the injector gap. However, unlike those earlier studies, we directly impose the injector current using experimental data, instead of coupling to an external capacitor bank model. Consequently, the injector voltage in our computations is solely a product of the evolution: it does not feed back into the current drive. Therefore, we are able to use the injector voltage as a diagnostic tool to make direct comparisons between the experiment and our calculations.

The resistivity is enhanced along the injector gap in order to encourage expansion of the flux bubble into the domain. Typically, the magnetic induction equation in NIMROD treats the entire domain surface as an idealized conductor, though steady-state soaking flux is permitted. While this assumption is reasonable for the inner and outer conducting surfaces on the time scales being considered, the injector gap is highly resistive, closer in practice to an idealized insulator than an idealized conductor. Without any enhancement of the resistivity along the injector, a significant fraction of the injected current "short-circuits" directly across the injector gap.

Ideally, the Lorentz force would pull the magnetic fieldlines away from the injector gap, resulting in the typical injector flux bubble expansion. However, the normal component of the flow velocity is constrained to zero along the entire domain boundary in the computations, which likewise constrains field-lines at the injector gap. Both past and current solutions have sought to avoid imposing some possibly unphysical or inconsistent boundary condition on the flow velocity, and therefore, mass fueling rate.

The previous numerical studies of SSPX discouraged current from flowing across the injector gap by imposing a uniform, cold plasma temperature in the row of elements along the injector at each time step in the computations. As the computations employed temperature-dependent Spitzer resistivity, the colder row of elements along the injector were significantly more resistive than those above. However, we chose not to employ this method for our study because unlike imposing some fixed boundary condition, it effectively discards some portion of the thermal energy in the interior of the domain in a manner not wholly consistent with the specified transport model.

Instead, we elongated the injector region by $10 \mathrm{~cm}$ and imposed an artificial enhancement to the resistivity along the injector gap, shown in Fig. 2. The enhanced resistivity only acts on the magnetic induction equation, which uses the effective resistivity $\eta_{\text {eff }}=\eta_{\text {plasma }}+\left(D_{s}-1\right) \eta_{i n j}$. This implementation allows us to impede current flow along the injector gap without affecting density, momentum, or thermal transport, except as they normally couple to the magnetic induction equation.

\section{COMPUTATIONAL RESULTS AND DISCUSSION}

In this section, we describe the results of our multi-pulse spheromak computations with the dual purposes of providing new insights into multi-pulse operation and demonstrating the effectiveness of our numerical model. Where practical,

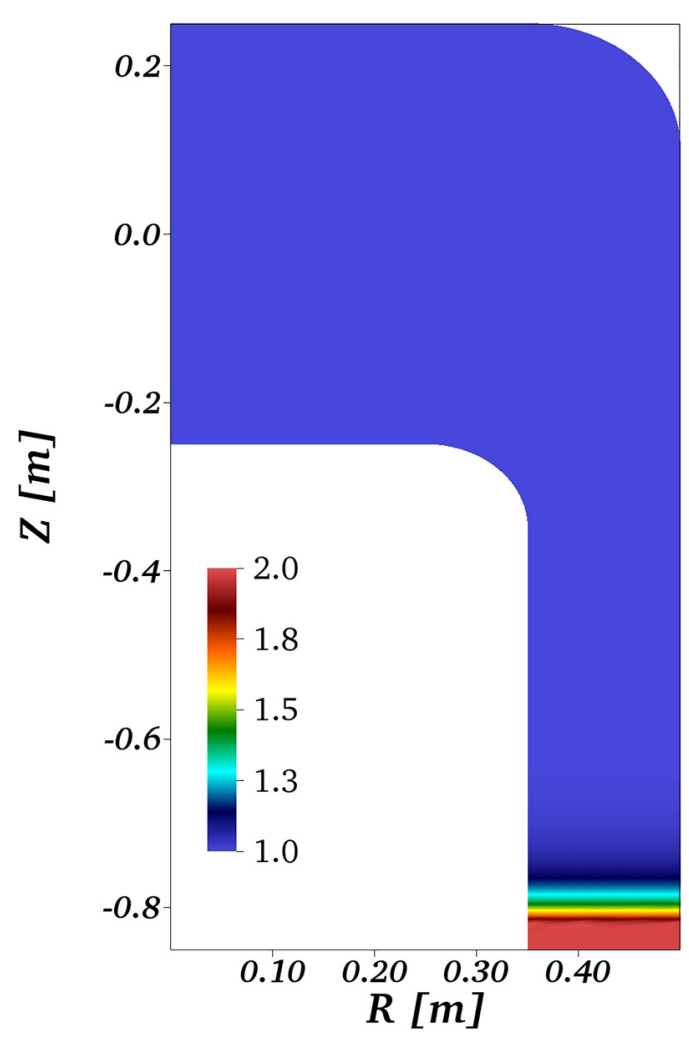

FIG. 2. The artificial enhancement to the resistivity is localized to the injector gap region. Color contours show the dimensionless diffusivity shaping profile $D_{s}$ applied to the magnetic induction equation.

we employ direct quantitative comparisons between synthetic diagnostics and experimental measurements in order to bolster our argument that our numerical model is suitable for characterizing plasma dynamics during multi-pulse operation of a CHI spheromak. For the experimental shots we are exploring with our computations, the capacitor bank was configured such that the first pulse of injector current-also referred to as the formation pulse- had a higher peak current and shorter duration than the subsequent "refluxing" pulses. First, we describe the evolution over multiple injector pulses, using the injector current and voltage traces for reference. Where appropriate, we reference Sec. I A and prior studies. Then, we compare the computational results of two shots in SSPX with similar $\lambda_{i n j}$ traces: shot \#19719 and shot \#19766. Reference 7 contains experimental measurements of magnetic fluctuations and electron transport for SSPX shot \#19719.

While there is qualitative agreement between the shape and relative amplitude of the experimental injector voltage and computational diagnostic over the course of a multipulse discharge, shown in Fig. 3, we cannot achieve generic quantitative agreement between the two, because of limitations of our numerical model in Sec. II. First, the electric field in our computations cannot accurately model sheath effects, which are likely to impact the impedance of the plasma-injector circuit. The resulting computational injector voltage primarily measures the stretching of magnetic fieldlines, an inductive effect. We also artificially enhance the resistivity along the injector gap to encourage expansion of the injector flux bubble. As long as the numerical value is at 


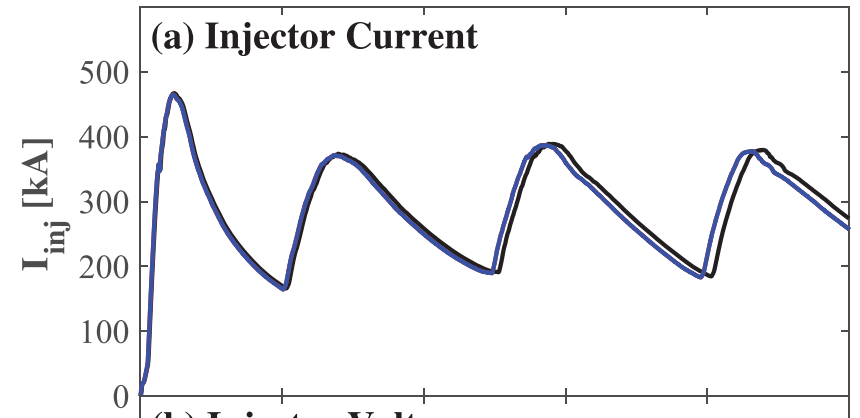

(b) Injector Voltage

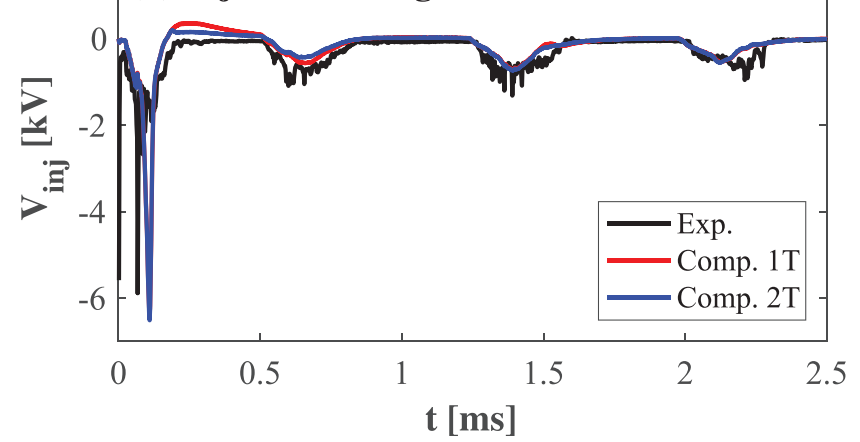

FIG. 3. Both the single and two temperature models produce qualitatively similar injector voltage traces as the experiment. Note that due to a transcription error, the injector current traces in the computations proceed approximately two percent faster than in SSPX shot \#19719. For simulations of SSPX shot \#19766, we had direct access to the experimental data, so there was no temporal discrepancy.

least as large as the plasma resistivity at the cold initial temperature, its value does not affect the qualitative shape of the injector voltage trace, though the magnitude does weakly scale with its value. Even though the model can be tuned to produce quantitative agreement between the experimental injector voltage and computational diagnostic, it probably is not necessary as qualitative agreement should be sufficient for our exploration of $\mathrm{CHI}$ spheromaks.

As shown in Fig. 3, the magnitude of the injector voltage correlates with the injector current, but it does not strongly correlate with reconnection during the column mode instability. The magnitude of the injector voltage is significantly greater during the first injector pulse-long before the onset of the column mode instability-than any subsequent pulse. For our simulated SSPX shot \#19719, we do not observe evidence of the column mode instability until the third injector pulse. We are not rejecting the correlation reported in Ref. 9 but merely suggest that the injector voltage is a poor diagnostic for determining the onset of the column mode instability during multi-pulse operation. The cyclical nature of the injected current during multi-pulse shots causes repeated expansion and contraction of the injector flux bubble and central spheromak, and the resulting stretching of the magnetic field lines dominates the computational-and likely, the experimental-injector voltage. Consequently, the small injector voltage perturbation produced by reconnection is overshadowed by the cyclical shot evolution.

During the formation pulse, i.e., the first pulse of injector current, a magnetic flux bubble forms along the injector gap. As the injector current increases, the flux bubble is ejected out of the injector region and expands into the flux conserver, as shown in Fig. 4. This evolution is consistent with that described in the experimental studies reported in Ref. 10 and previous modeling reported in Ref. 9. The flux bubble continues expanding until shortly after the injector current begins decreasing. As the injector current decreases, the flux bubble partially recedes into the injector region. High current density at the separatrix (Fig. 5) is consistent with the occurrence of reconnection that detaches the central spheromak plasma from the injector. The reconnection is similar to pull-type reconnection in the magnetic reconnection experiment when the poloidal field current decreases. ${ }^{24}$

The evolution during the first refluxing pulse, i.e., the second pulse of injector current, is similar to that during the formation pulse: A magnetic flux bubble forms near the injector gap and expands until coming into contact with the central spheromak plasma remaining from the formation
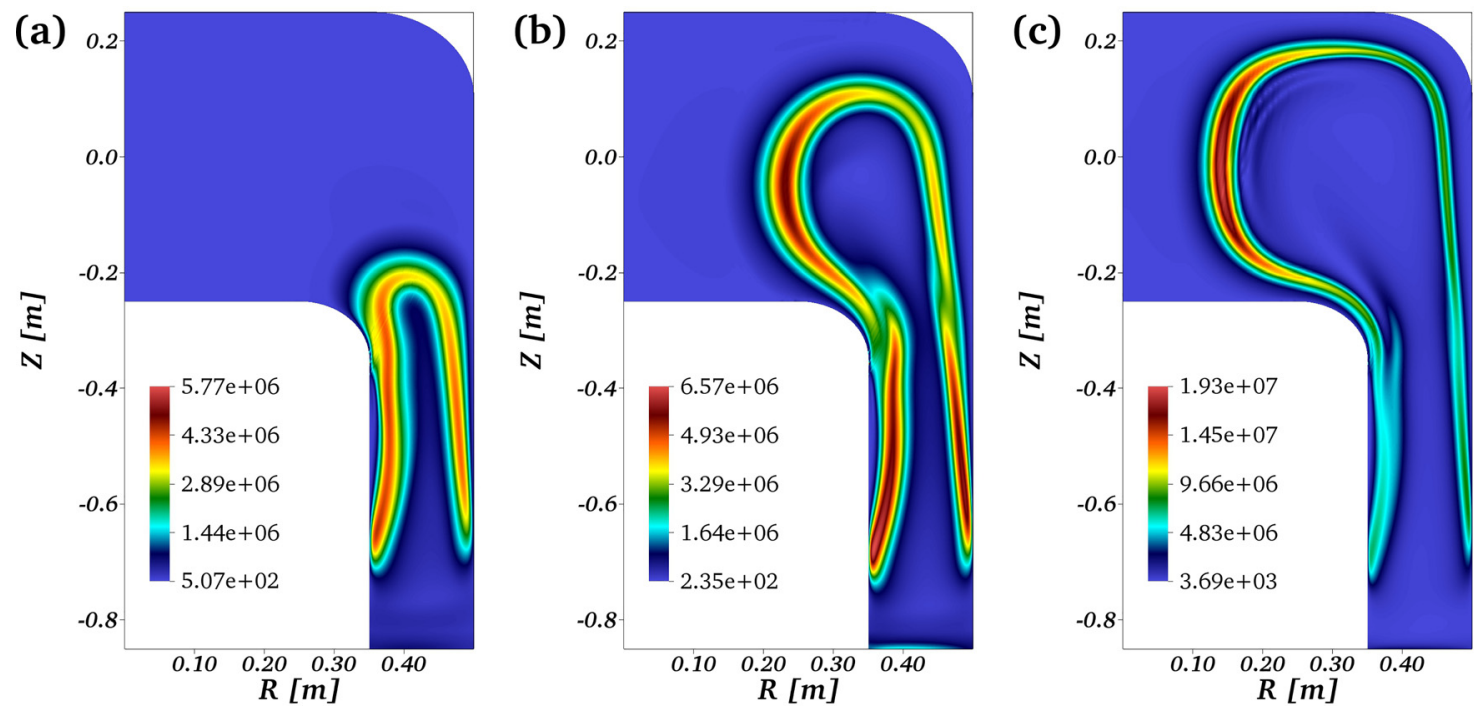

FIG. 4. The expansion of the injector flux bubble is consistent with experimental discharges in SSPX and the results of prior computational studies. Color contours of total current density in units of $\mathrm{A} / \mathrm{m}^{2}$ are shown for the following times: (a) $t=80 \mu \mathrm{s}$, (b) $t=97 \mu \mathrm{s}$, and (c) $t=116 \mu \mathrm{s}$. Simulated SSPX shot \#19719, single temperature model. 

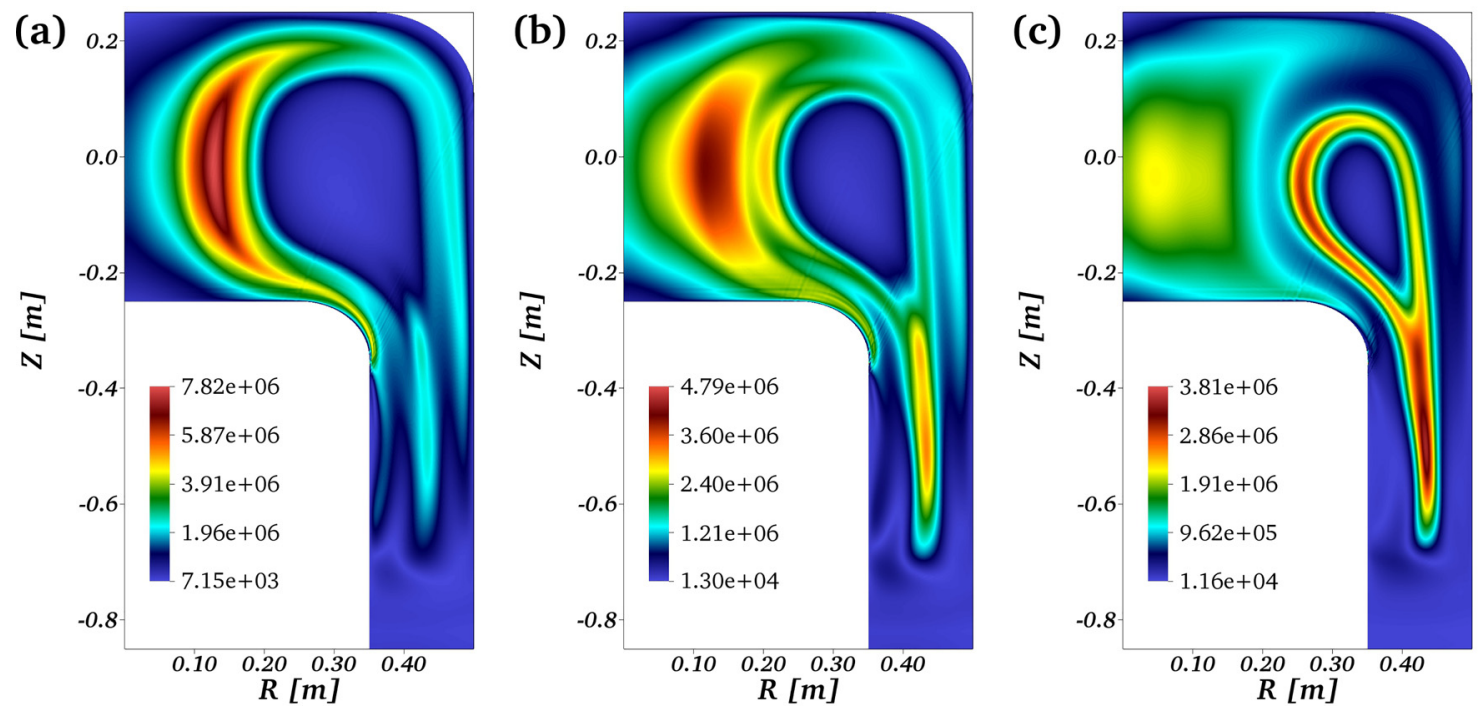

FIG. 5. As the current decreases at the end of an injector pulse, the spheromak plasma recedes into the throat of the injector. The contraction of the plasma and high current density present at the separatrix indicate the occurrence of pull-type reconnection in the injector region. Color contours of current density in units of $\mathrm{A} / \mathrm{m}^{2}$ are shown for the following times (a) $t=270 \mu \mathrm{s}$, (b) $t=349 \mu \mathrm{s}$, and (c) $t=500 \mu \mathrm{s}$. Simulated SSPX shot \#19719, single temperature model.

pulse. The flux bubble reconnects with the edge of the central spheromak, which is once again line-tied to the injector. As shown by Fig. 6, the core of the central spheromak does not reconnect with the flux bubble and is compressed towards the top of the flux conserver during the rise in injector current. While the injector current declines at the end of the pulse, the plasma again partially recedes into the injector region. Reconnection occurs at the separatrix, and the central spheromak once again detaches from the injector.

The second reflux pulse, i.e., the third pulse of injector current, proceeds like the previous refluxing pulse until the onset of the column mode instability. We use edge magnetic diagnostics to determine the onset of the column mode instability because this method allows for direct comparison of experimental and synthetic magnetic diagnostic data, which is presented in Fig. 7. Spreading of the traces indicates the presence of non-axisymmetric MHD activity around $t=1.5 \mathrm{~ms}$. For the computation results, Figs. 7(b) and 7(c), the spreading begins around $t=1.35 \mathrm{~ms}$ and reaches its peak amplitude around $t=1.55 \mathrm{~ms}$.

Prior to the column mode instability, the plasma is predominantly axisymmetric, and the temperature and magnetic surfaces are well correlated. Shortly after the edge magnetic diagnostics detect the onset of the column mode, there is a noticeable non-axisymmetric perturbation to the predominantly toroidal spheromak, Fig. 8(a). As the mode grows in amplitude, the central current column kinks, which appears as tilting and bending of the "donut hole" in the center of Figs. 8(b) and 8(c). Near the peak mode amplitude, the temperature and magnetic surfaces decouple, as the temperature
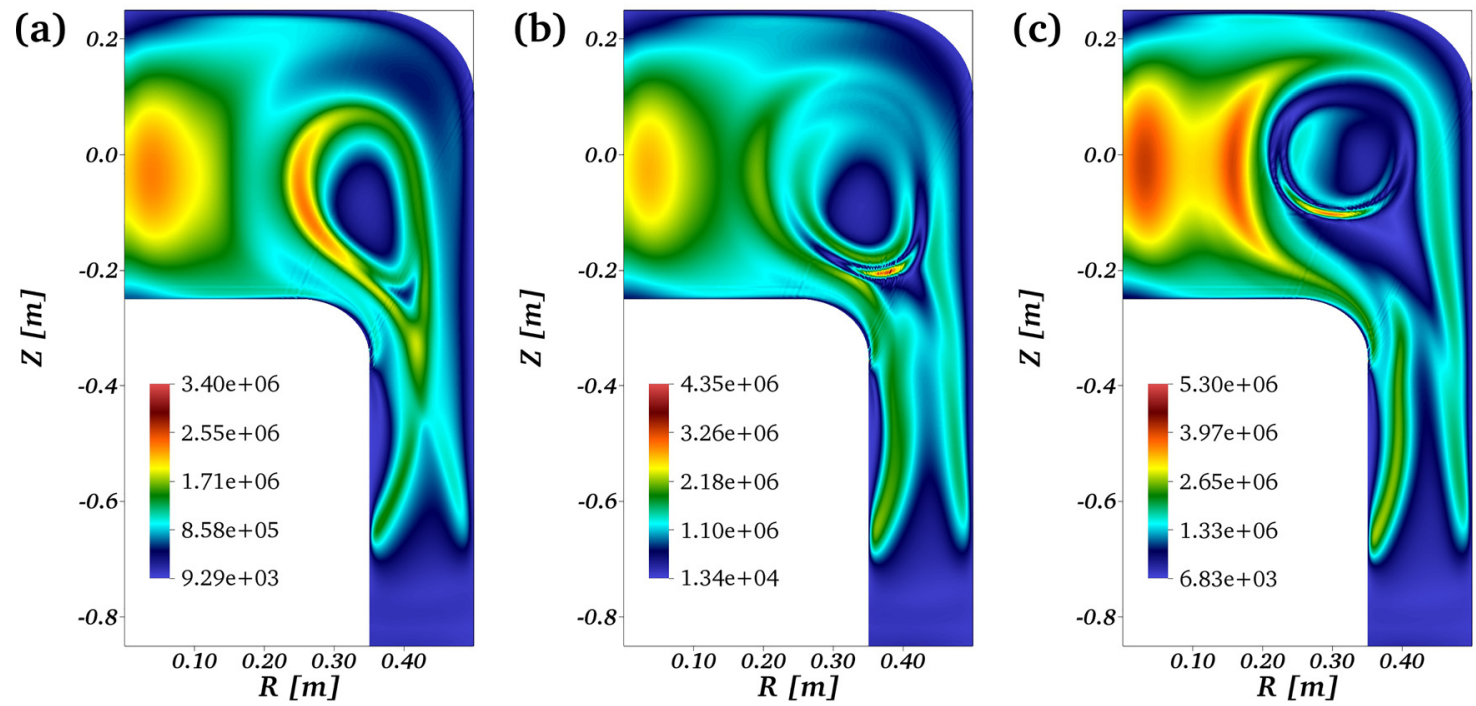

FIG. 6. During a refluxing pulse, a second expanding flux bubble forms in the injector region. When the flux bubble comes into contact with the pre-existing spheromak plasma, reconnection opens and connects the outer flux surfaces of the spheromak to the injector. During this process, the spheromak plasma is compressed into the flux conserver. Color contours of total current density in units of $\mathrm{A} / \mathrm{m}^{2}$ are shown for the following times: (a) $t=571 \mu \mathrm{s}$, (b) $t=619 \mu \mathrm{s}$, and (c) $t=684 \mu$ s. Simulated SSPX shot \#19719, single temperature model. 

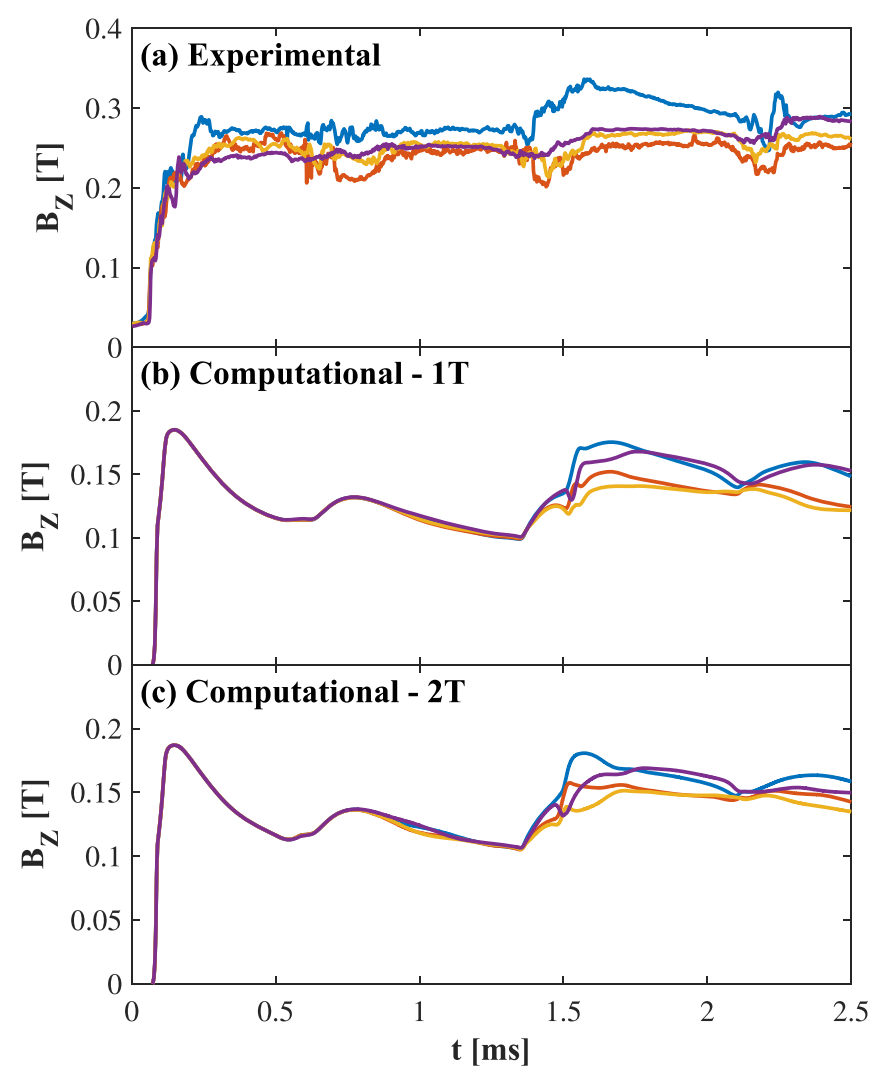

FIG. 7. Both experimental and synthetic diagnostics of the vertical magnetic field at the device mid-plane indicate the presence of a large, toroidally asymmetric mode at $t=1.55 \mathrm{~ms}$. All magnetic diagnostics are located at the same poloidal position, $R=49.9 \mathrm{~cm}, Z=-9.0 \mathrm{~cm}$. The different colored traces correspond to different toroidal positions. (a) The experimental magnetic diagnostics are located at $0^{\circ}, 90^{\circ}, 135^{\circ}$, and $270^{\circ}$. Both (b) the single temperature calculation and (c) the two temperature calculation have four magnetic diagnostics with the same toroidal spacing as the experiment.

profile rapidly equilibrates to fit the new magnetic topology. Similar evolution is reported in Ref. 25 during a single formation and sustainment pulse for both the experimental high-speed camera images and the corresponding isosurfaces of the simulated electron temperature.

As described in Sec. I A, the change in magnetic topology during the column mode instability redistributes the local current density producing significant poloidal flux amplification, Fig. 9(b). For this study, we employ a local measure of poloidal amplification, $A_{\Psi}$, which is the ratio of the maximum value of the poloidal flux at a given instant to the maximum value of the vacuum poloidal flux. A value of
$A_{\Psi}$ greater than unity necessarily indicates poloidal flux amplification. While an integral measure of poloidal amplification may be less sensitive to the current density distribution, leading to better quantitative agreement between the results of both numerical models, it would be far less useful for determining the onset of the column mode instability: The "average" poloidal flux would increase from expansion of the injector flux bubble, even without triggering the column mode instability.

Local poloidal flux amplification is observed around $t=1.5 \mathrm{~ms}$ in Fig. 7, which is after the onset and shortly before the peak amplitude of the non-axisymmetric MHD mode activity observed by the magnetic diagnostics. Both the single and two fluid-temperature models produced qualitatively similar results for localized poloidal flux amplification and total magnetic energy content. Quantitative differences between the two models can be explained by differences in the electron local temperature profile. As shown in Fig. 10, the two-temperature model produces significantly higher and more narrowly peaked electron temperature profiles. Consequently, the temperature dependence of electrical resistivity affects the local current distribution prior to and during the column mode instability, the resulting magnetic self-organization, and ultimately, the degree of local poloidal flux amplification.

The evolution during the third refluxing pulse, i.e., the fourth injector current pulse, proceeds similarly to the evolution during the first refluxing pulse: The central spheromak is compressed while the injector current is rising, but neither the magnetic diagnostics nor poloidal flux amplification indicates the column mode instability. The column mode instability does occur again over the interval $t=3-3.5 \mathrm{~ms}$ during fourth refluxing pulse, i.e., the fifth pulse of injector current. The resultant poloidal flux amplification is shown in Fig. 11.

Each computation employs a single physical model throughout the entire domain for all simulated time. However, there are significant qualitative differences in the local magnetic topology between reconnection during the column mode instability and reconnection during refluxing, i.e., between the injector flux bubble and the central spheromak, that affects how much magnetic energy is converted to kinetic and thermal energy. Reconnection during the column mode instability occurs with a comparatively strong guide-field at the separatrix, generated by the annular current column. As the current column kinks and rotates into the horizontal midplane, the current column
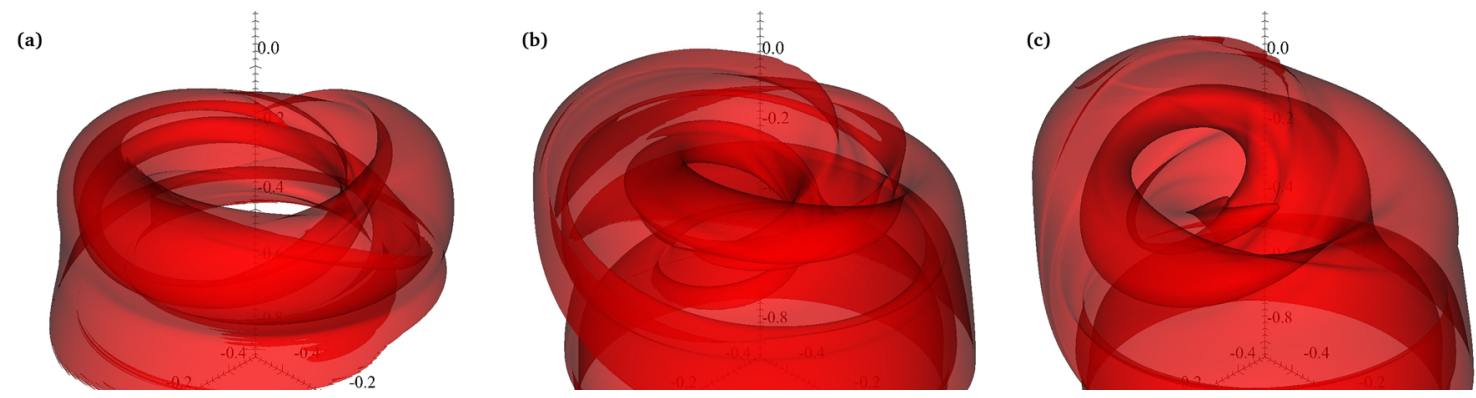

FIG. 8. Translucent contours of electron temperature $\left(T_{e}=21 \mathrm{eV}\right.$ ) at (a) $t=1.379 \mathrm{~ms}$, (b) $t=1.459 \mathrm{~ms}$, and (c) $t=1.501 \mathrm{~ms}$ show the change in plasma topology over the column mode instability. Simulated shot \#19719, two temperature model. 


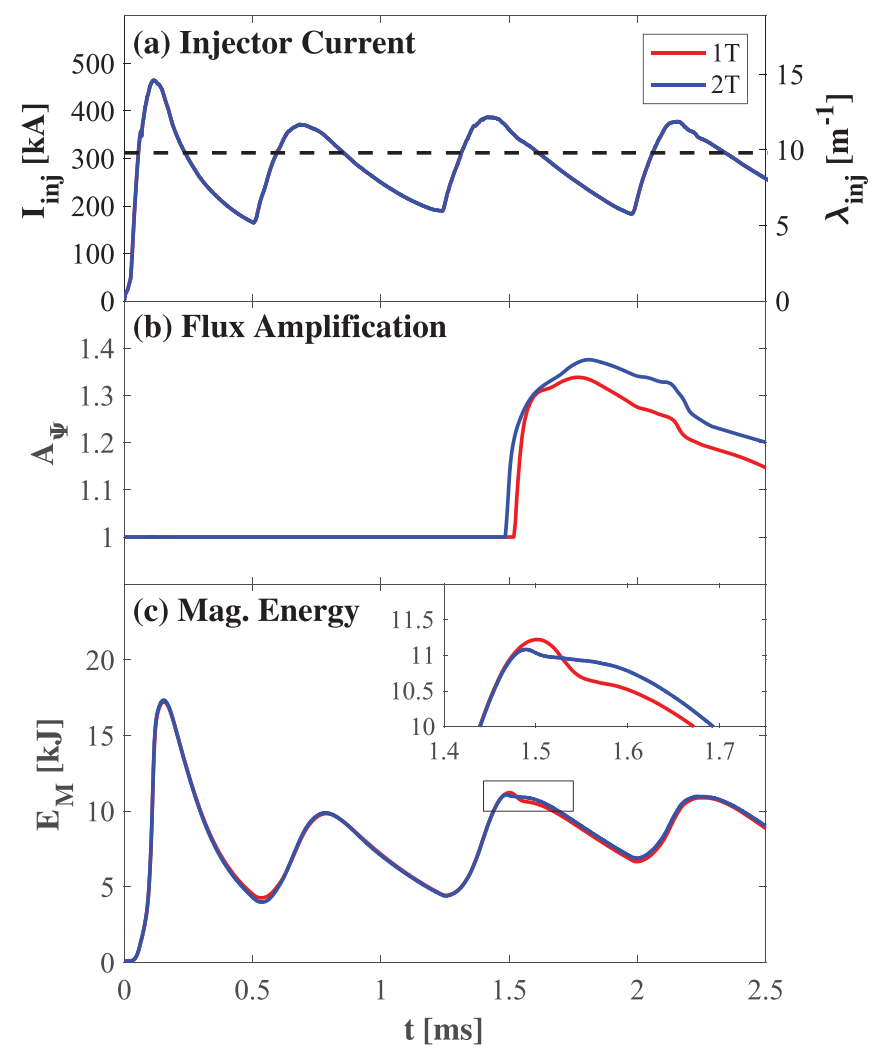

FIG. 9. Although the injected current (a) and corresponding $\lambda_{i n j}$ periodically exceed the eigenvalue (Taylor-state) $\lambda_{e}=9.8 \mathrm{~m}^{-1}$ for the SSPX flux conserver-indicated by the dashed line-poloidal flux amplification (b) does not occur until $t \simeq 1.5 \mathrm{~ms}$, just prior to the peak amplitude of the magnetic mode. The total magnetic energy (c) closely follows the injected current. Even though the kink instability leads to large-scale change of the magnetic topology, the amount of magnetic energy converted by reconnection is small as shown in the figure (c) inset. Both the single and two temperature models produce qualitatively similar results though the two temperature model produces significantly more localized poloidal flux amplification.

projects its (local magnetic-coordinate) axial field onto the global toroidal field, i.e., the guide-field with respect to the orientation of the separatrix. Consequently, reconnection during the column mode produces a significant drop in the total magnetic energy content of the plasma not observed during reconnection between an injector flux bubble and a central spheromak plasma.

The maximum discrepancy in the total magnetic energy is less than $5 \%$ between the single and two fluid-temperature models. The traces are most dissimilar during the column mode around the time at which the peak mode amplitude occurs, as seen in the inset of Fig. 9(c).

While the onset of the column mode kink instability is predicted by linear stability theory, the conditions in the resulting spheromak plasma, e.g., the degree of poloidal flux amplification, are set by the dynamic, nonlinear evolution of the spheromak plasma. To explore how nonlinear spheromak evolution is affected by the injected current and magnitude of the bias flux, we compare the simulated results of two SSPX discharges with similar $\lambda_{i n j}$ traces: shots \#19719 and \#19766, which, respectively, have 40 and $60 \mathrm{mWb}$ of bias flux in the modified flux configuration.

The initial column mode kink instability occurs during the third injector current pulse for shot \#19719 and during the second injector current pulse for shot \#19766. For shot \#19766, the poloidal flux amplification produced by the initial kink instability is anemic $(A \Psi<1.1)$ compared to that produced by any other kink in either discharge. The third and subsequent injector current pulses for both discharges have similarly shaped poloidal flux traces, but shot \#19766 has consistently greater poloidal flux amplification $\left(\Delta A_{\Psi} \geq 0.3\right)$ than shot \#19719. Both the earlier occurrence of the column mode instability and greater poloidal flux amplification for shot \#19766 are consistent with the larger bias flux producing an increase in the free energy available to the kink instability: The Lorentz force acting on the annular current column increases with the bias flux. Therefore, the increase in bias flux has not fundamentally changed the plasma evolution but rather has made the column mode instability more dynamically accessible.

To allow for direct comparisons between the discharges, we normalize the magnetic helicity content $K=\int_{V} \mathbf{A} \cdot \mathbf{B} d^{3} \mathbf{x}$ to the square of the nominal bias flux. For any two magnetic field configurations with well-defined vector potential fields and related by a single constant scalar multiplier $\alpha$, i.e., (a)

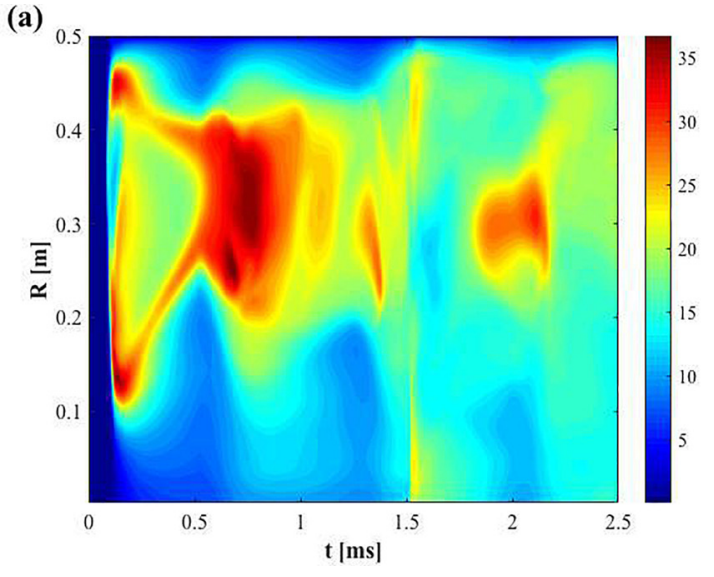

(b)

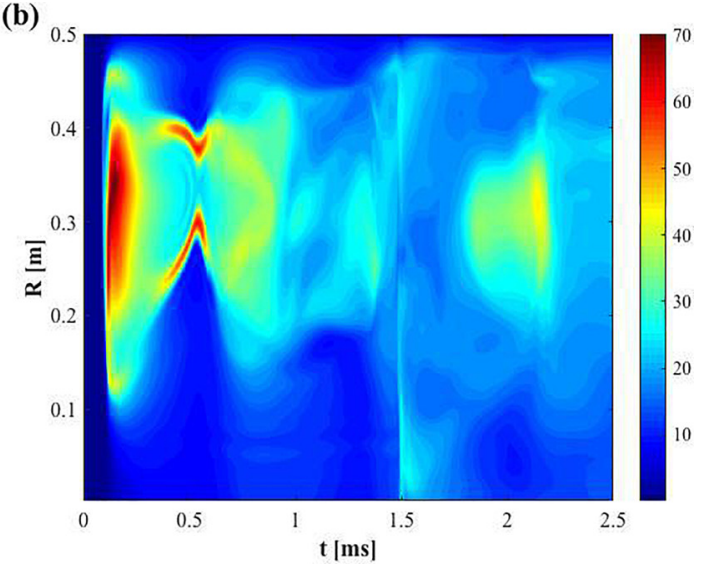

FIG. 10. The single and two temperature models produce very similar magnetic evolution despite very different temperature profiles. Chordal measurements of the electron temperature in $\mathrm{eV}$ at $Z=0, \phi=0$ are shown for simulations of SSPX shot \#19719 evolved with (a) a single fluid temperature model and (b) a two fluid temperature model. The two-temperature model produces significantly higher and more narrowly peaked electron temperature profiles. 

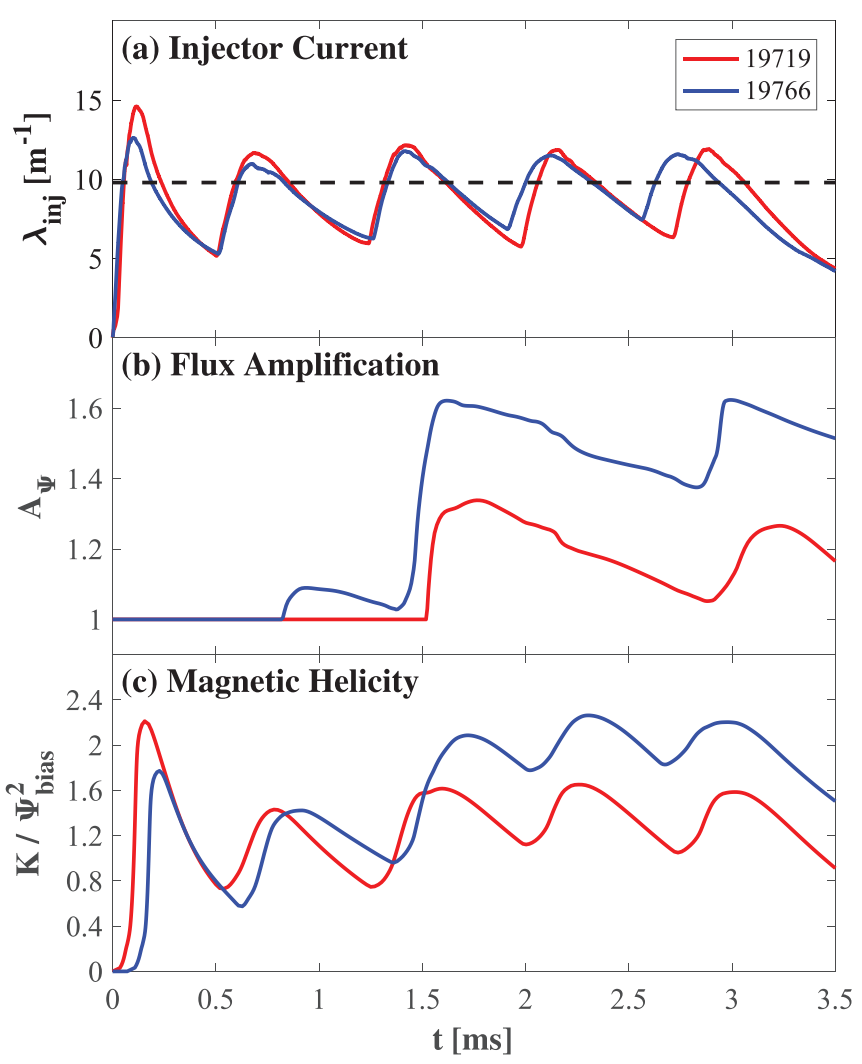

FIG. 11. Driven by similar $\lambda_{i n j}$ traces, SSPX shots \#19719 and \#19766 produced similarly shaped traces for poloidal flux amplification and magnetic helicity content. For SSPX shots \#19719 and \#19766, the maximum injected currents were $465 \mathrm{kA}$ and $602.4 \mathrm{kA}$, respectively.

$\mathbf{B}_{1}=\alpha \mathbf{B}_{0}$, the magnetic helicity content is related by $K_{1}=\alpha^{2} K_{0}$. Here, we use the nominal bias flux as an analogue to $\alpha$ because a true constant scalar multiplier would produce perfectly overlapping traces of $K / \Psi_{\text {bias }}^{2}$. The discharges produce similar, but not identically, shaped traces of normalized magnetic helicity.

During current injection, open magnetic field lines connect the injector and the edge of the spheromak plasma. As the injector current rises, expanding magnetic flux from the injector region carries magnetic helicity into the flux conserver. Likewise, as the injector current decreases, contraction of the magnetic flux into the injector region pulls magnetic helicity out of the flux conserver. Hence, the similar shape between traces of injected current and magnetic helicity content is shown in in Fig. 11. The change in the magnetic topology produced by the column mode instability decouples some of the magnetic helicity content from the injector. The "confinement" of the magnetic helicity content in the core spheromak plasma is then dominated by resistive diffusion of the toroidal current produced by the column mode instability.

As the injector current decreases at the end of a pulse, resistive decay of the toroidal current near the magnetic axis causes our measure of poloidal flux amplification $A_{\Psi}$ to decrease. During the third pulse of injector rates, $A_{\Psi}$ decreases slightly more slowly with a higher bias flux. The difference in rates is more pronounced during the fifth pulse of injector current. After the occurrence of the column mode instability, the magnetic helicity content of the system increases, including both the maximum following each pulse of injector current and the minimum preceding the next pulse. The formation pulse of shot \#19766 produces significantly less normalized magnetic helicity content than the formation pulse of shot \#19719, which is expected as the peak injector lambda for each injector current pulse in shot $\# 19766$ is consistently lower than the corresponding peak in shot \#19719. The meager poloidal flux amplification during the second injector current pulse in shot \#19766 confines enough of its magnetic helicity content that the second peak is the approximately the same for both discharges, despite the differences in injector lambda. All the remaining peaks for shot \#19766 have significantly more normalized magnetic helicity content than the corresponding peaks for shot \#19719, so much so that even the minimum following the third and fourth pulses of shot \#19766 is greater than the maximum for the third, fourth, and fifth pulses of shot \#19719.

In Fig. 11, there is a variable time-lag between traces of the injector current and magnetic helicity content, which corresponds to the magnetic diffusion time for injector flux to penetrate into the flux conserver or "core" spheromak plasma. The time-lag between the rise in injector current and magnetic helicity at the beginning of an injector current pulse is much shorter than the time-lag between when the injector current and magnetic helicity reach their maximum for the pulse. The time-lag is also consistently longer for shot \#19766, i.e., the shot with higher bias flux. The difference in time-lags between shots is most obvious for the second and third injector pulses, where the injector current begins to increase and reaches its peak value at approximately the same time, but the corresponding rise and peak for magnetic helicity content occurs $\sim 100 \mu$ s later in shot $\# 19766$ than shot \#19719. For shot \#19719, the time-lag is approximately $10-50 \mu$ s at the beginning of each pulse and $100-200 \mu$ s at its peak. For shot \#19766, the time-lag is approximately $100-120 \mu \mathrm{s}$ at the beginning of each pulse and $200-300 \mu$ s at its peak.

The time-lag at the beginning of a pulse corresponds to the time required for an injector flux bubble to form and begin expanding towards the mouth of the injector, after which the magnetic helicity content of the system begins to gradually increase. Once the injector flux bubble begins reconnecting with the central spheromak plasma, the rate at which magnetic helicity is injected into the system greatly increases. The time-lag at a pulse peak corresponds to the magnetic diffusion time between the current-carrying magnetic field lines tied to the injector and the "core" spheromak plasma. Here, we consider our core spheromak plasma to include both regions of closed magnetic field lines and regions where the magnetic field lines persist for many transits around the central spheromak such that the effective parallel thermal transport is much smaller than cross-field transport. As shot \#19766 achieves higher temperature-and therefore, has a much longer resistive diffusion time- than shot \#19719, we expect the lag-time to be greater. While the time-lag correlates with the plasma temperature, it does not directly affect the kink instability. 
The lag-time in our computations is consistent with experimental observations from an earlier set of SSPX multi-pulse shots (\#16208-\#16210), reported in Ref. 26. The shots had $28 \mathrm{mWb}$ of bias flux in the modified flux configuration and a peak $\lambda_{i n j}=1.2 \mathrm{~m}^{-1}$, i.e., less than one-tenth of the peak $\lambda_{i n j}$ for shot $\# 19719$ and \#19766. Using an insertable magnetic probe, experimentalists measured a time lag in the response between regions of open and closed magnetic flux at the beginning of each pulse. The lag-time was consistent with estimates of the magnetic diffusion time. There was a similar time-lag for the magnetic helicity content-determined through a series of CORSICA equilibria reconstructions- to increase by the square of the average flux at the beginning of the pulse.

\section{SUMMARY AND CONCLUSIONS}

In this paper, we used nonlinear, numerical computation with the NIMROD code to reproduce the global magnetic evolution of a CHI spheromak during multi-pulse operation. Our computations predict the same onset for the model mode instability as experimental magnetic diagnostics, e.g., the column mode first occurs during the third injector pulse of SSPX shot \#19719. The diagnostics in Figs. 7 and 9 show little difference between the single- and two-temperature computations despite Fig. 10 showing significant quantitative and qualitative differences in the electron temperature profile, and correspondingly, the resistivity. Thus, the global magnetic evolution in our computations is fairly robust to changes in the electron temperature profile produced with different thermal transport models. Therefore, we can conclude that the more computationally efficient single fluidtemperature model is sufficient for qualitatively assessing spheromak performance in our future numerical studies. While impurity ion measurements suggest that $T_{i} \sim T_{e}$ during the quiescent phase between pulses, they also suggest $T_{i}>T_{e}$ during the kink instability, ${ }^{27}$ so this result is significant. A more complete transport model is then needed to quantify performance gains.

Our numerical model achieves good agreement between the computational injector voltage and experimental measurements from the corresponding SSPX discharges, despite only specifying the injector current trace. The agreement between the experimental and computational injector voltage will allow us to study the dynamics in a desired parameter regime, while simultaneously estimating the injector voltage required to produce the injector current trace. Then, we can assess whether the corresponding experimental design is technologically feasible, particularly with regards to the minimum capacitor bank requirements. Likewise, we can consider the marginal cost for incremental improvements in both the design and operating parameters.

The magnitude of the peak injector voltage during the formation pulse greatly exceeds that of any subsequent refluxing pulse. Our model lacks sheath effects, so the dominant contribution to the injector voltage for both the experiment and computations is the inductive stretching of magnetic field lines during expansion of the injector flux bubble. After reconnection between the expanding flux bubble and central spheromak during a refluxing pulse, the plasma configuration greatly resembles that during the initial formation pulse, except there is a central spheromak surrounded by a current layer attached to the injector, instead of just the current layer. Refluxing causes the central spheromak to slightly compress towards the geometric axis, which is likely to be beneficial for driving the column mode instability. The overall shape of the injector voltage trace correlates well with the injector current, though there is a (much smaller) response from the column mode kink instability.

The column mode instability does not necessarily occur during each pulse of injector current, even when the injector lambda transiently crosses the steady-state threshold for the linear kink instability. Each pulse of injector current produces, at most, a single impulsive increase in the poloidal flux amplification, which corresponds to a single large kink instability: the injector current is not sustained above the threshold for the kink instability for sufficiently long to the observe numerous smaller kinks expected to occur after the initial kink. Between pulses, the injector current drops below the threshold for the kink instability long enough for the system to relax toward an axisymmetric equilibrium state, i.e., significant decay of any toroidal asymmetries produced by the column mode instability.

Our simulations of two SSPX shots (\#19719 and \#19766) with similar injector lambda traces produced qualitatively similar spheromak evolution. Concurrently increasing the magnitude of the bias flux and the peak injected current significantly increases the Lorentz force acting on the annular current column, and therefore, the free energy available to the kink instability. Consequently, this increases the poloidal flux amplification produced by the column mode instability and the magnetic helicity content in the resulting spheromak. In the paper to follow, we will conduct a more thorough exploration of how various externally controllable parameters, e.g., injected current and bias flux, affect the spheromak performance during the initial formation pulse.

\section{ACKNOWLEDGMENTS}

This work is supported by the Defense Advanced Research Projects Agency (DARPA) under Grant No. N66001-14-1-4044.

Access to Sustained Spheromak Physics eXperiment (SSPX) data is provided under the auspices of Lawrence Livermore National Laboratory (LLNL) under Contract No. DE-AC52-07NA27344. Special thanks to Harry McLean and Bill Meyer for their support.

Computations have been performed at the following facilities:

- The UMBC High Performance Computing Facility (HPCF). The facility is supported by the U.S. National Science Foundation through the MRI program (Grant Nos. CNS-0821258 and CNS-1228778) and the SCREMS program (Grant No. DMS-0821311), with additional substantial support from the University of Maryland, Baltimore County (UMBC). See www.umbc.edu/hpcf for more information on HPCF and the projects using its resources. 
- The U.S. Army Engineer Research and Development Center (ERDC) DoD Supercomputing Resource Center (DSRC), which is operated by the DoD High Performance Computing Modernization Program (HPCMP).

- The National Energy Research Scientific Computing Center, which is supported by the Office of Science of the U.S. Department of Energy under Contract No. DE-AC0205CH11231.

${ }^{1}$ M. Yamada, R. Kulsrud, and H. Ji, "Magnetic reconnection," Rev. Mod. Phys. 82, 603-664 (2010).

${ }^{2}$ E. B. Hooper, R. H. Bulmer, B. I. Cohen, D. N. Hill, C. T. Holcomb, B. Hudson, H. S. McLean, L. D. Pearlstein, C. A. Romero-Talamás, C. R. Sovinec, B. W. Stallard, R. D. Wood, and S. Woodruff, "Sustained spheromak physics experiment (SSPX): design and physics results," Plasma Phys. Controlled Fusion 54(11), 113001 (2012).

${ }^{3}$ P. M. Bellan, Spheromaks: A Practical Application of Magnetohydrodynamic Dynamos and Plasma Self-Organization (Imperial College Press, 2000).

${ }^{4}$ S. Woodruff, J. E. Stuber, C. Bowman, P. E. Sieck, P. A. Melnik, C. A. Romero-Talamás, J. B. O’Bryan, and R. L. Miller, “Adiabatic compression of a compact torus," Fusion Sci. Technol. 72(4), 705-712 (2017).

${ }^{5}$ T. K. Fowler, R. Jayakumar, and H. S. McLean, "Stable spheromaks sustained by neutral beam injection," J. Fusion Energy 28(1), 118-123 (2009).

${ }^{6}$ T. R. Jarboe, B. S. Victor, B. A. Nelson, C. J. Hansen, C. Akcay, D. A. Ennis, N. K. Hicks, A. C. Hossack, G. J. Marklin, and R. J. Smith, "Imposed-dynamo current drive," Nucl. Fusion 52(8), 083017 (2012).

${ }^{7}$ B. Hudson, R. D. Wood, H. S. McLean, E. B. Hooper, D. N. Hill, J. Jayakumar, J. Moller, D. Montez, C. A. Romero-Talams, T. A. Casper, J. A. Johnson, L. L. LoDestro, E. Mezonlin, and L. D. Pearlstein, "Energy confinement and magnetic field generation in the SSPX spheromak," Phys. Plasmas 15(5), 056112 (2008).

${ }^{8}$ C. R. Sovinec, D. D. Schnack, A. Y. Pankin, D. P. Brennan, H. Tian, D. C. Barnes, S. E. Kruger, E. D. Held, C. C. Kim, X. S. Li, D. K. Kaushik, S. C. Jardin, and NIMROD Team. "Nonlinear extended magnetohydrodynamics simulation using high-order finite elements," J. Phys.: Conf. Ser. 16(1), 25 (2005).

${ }^{9}$ E. B. Hooper, B. I. Cohen, H. S. McLean, R. D. Wood, C. A. RomeroTalamas, and C. R. Sovinec, "Nimrod resistive magnetohydrodynamic simulations of spheromak physics," Phys. Plasmas 15(3), 032502 (2008).

${ }^{10}$ E. B. Hooper, "Physics issues of a spheromak refluxing scenario," Plasma Phys. Controlled Fusion 53(8), 085008 (2011).

${ }^{11}$ T. R. Jarboe, "Review of spheromak research," Plasma Phys. Controlled Fusion 36(6), 945 (1994).

${ }^{12}$ R. D. Wood, D. N. Hill, E. B. Hooper, S. Woodruff, H. S. McLean, and B. W. Stallard, "Improved operation of the SSPX spheromak," Nucl. Fusion 45(12), 1582 (2005).
${ }^{13}$ C. A. Romero-Talamás, C. Holcomb, P. M. Bellan, and D. N. Hill, "Spheromak formation and sustainment studies at the sustained spheromak physics experiment using high-speed imaging and magnetic diagnostics," Phys. Plasmas 13(2), 022502 (2006).

${ }^{14}$ J. B. O'Bryan, C. R. Sovinec, and T. M. Bird, "Simulation of currentfilament dynamics and relaxation in the Pegasus spherical tokamak," Phys. Plasmas 19(8), 080701 (2012).

${ }^{15}$ C. R. Sovinec, J. M. Finn, and D. del Castillo-Negrete, "Formation and sustainment of electrostatically driven spheromaks in the resistive magnetohydrodynamic model," Phys. Plasmas 8(2), 475-490 (2001).

${ }^{16}$ J. B. O'Bryan and C. R. Sovinec, "Simulated flux-rope evolution during non-inductive startup in Pegasus," Plasma Phys. Controlled Fusion 56(6), 064005 (2014)

${ }^{17}$ C. R. Sovinec, T. A. Gianakon, E. D. Held, S. E. Kruger, D. D. Schnack, and NIMROD Team, "Nimrod: A computational laboratory for studying nonlinear fusion magnetohydrodynamics," Phys. Plasmas 10(5), 1727-1732 (2003).

${ }^{18}$ D. Brennan, P. K. Browning, R. A. M. Van der Linden, A. W. Hood, and S. Woodruff, "Stability studies and the origin of the $n=1$ mode in the SPHEX spheromak experiment," Phys. Plasmas 6(11), 4248-4259 (1999).

${ }^{19}$ C. R. Sovinec, A. H. Glasser, T. A. Gianakon, D. C. Barnes, R. A. Nebel, S. E. Kruger, D. D. Schnack, S. J. Plimpton, A. Tarditi, and M. S. Chu, "Nonlinear magnetohydrodynamics simulation using high-order finite elements," J. Comput. Phys. 195(1), 355-386 (2004).

${ }^{20}$ C. R. Sovinec, B. I. Cohen, G. A. Cone, E. B. Hooper, and H. S. McLean, "Numerical investigation of transients in the SSPX spheromak," Phys. Rev. Lett. 94(3), 035003 (2005).

${ }^{21}$ B. I. Cohen, E. B. Hooper, R. H. Cohen, D. N. Hill, H. S. McLean, R. D. Wood, S. Woodruff, C. R. Sovinec, and G. A. Cone, "Simulation of spheromak evolution and energy confinement," Phys. Plasmas 12(5), 056106 (2005).

${ }^{22}$ S. I. Braginskii, "Transport processes in a plasma," Rev. Plasma Phys. 1, 205-311 (1965).

${ }^{23}$ R. Courant, K. Friedrichs, and H. Lewy, "On the partial difference equations of mathematical physics," IBM J. Res. Dev. 11(2), 215-234 (1967).

${ }^{24}$ M. Yamada, "Review of controlled laboratory experiments on physics of magnetic reconnection," J. Geophys. Res.: Space Phys. 104(A7), 14529-14541, https://doi.org/10.1029/1998JA900169 (1999).

${ }^{25}$ B. I. Cohen, C. A. Romero-Talams, D. D. Ryutov, E. B. Hooper, L. L. LoDestro, H. S. McLean, T. L. Stewart, and R. D. Wood, "The role of the $\mathrm{n}_{\phi}=1$ column mode in spheromak formation," Phys. Plasmas 16(4), 042501 (2009).

${ }^{26}$ C. A. Romero-Talamás, E. B. Hooper, R. Jayakumar, H. S. McLean, R. D. Wood, and J. M. Moller, "Measurements and phenomenological modeling of magnetic flux buildup in spheromak plasmas," Phys. Plasmas 15(4), 042503 (2008)

${ }^{27}$ J. D. King, H. S. McLean, R. D. Wood, C. A. Romero-Talamás, J. M. Moller, and E. C. Morse, "An ion Doppler spectrometer instrument for ion temperature and flow measurements on SSPX," Rev. Sci. Instrum. 79(10), 10F535 (2008). 